\title{
Microbunching instability in storage rings: Link between phase-space structure and terahertz coherent synchrotron radiation radio-frequency spectra
}

\author{
E. Roussel, C. Evain, ${ }^{*}$ C. Szwaj, and S. Bielawski \\ Laboratoire de Physique des Lasers, Atomes et Molécules, UMR CNRS 8523, \\ Centre d'Études et de Recherches Lasers et Applications, FR CNRS 2416, \\ Université des Sciences et Technologies de Lille, F-59655 Villeneuve d'Ascq Cedex, France
}

(Received 12 April 2013; published 13 January 2014)

\begin{abstract}
In storage rings, when the number of electrons in a bunch exceeds a threshold value, microstructures appear spontaneously in the bunch. Experimental observations of these microstructures and their evolution are indirect to obtain. In practice, most information is obtained from the terahertz coherent synchrotron radiation (THz CSR), since microstructures in the millimeter range radiate $\mathrm{THz} \mathrm{CSR}$. Here we show, thanks to a simple analytical formula associated to numerical simulations, that a frequency component in the $\mathrm{THz}$ CSR signal permits one to obtain information about the structure of the electron bunch longitudinal phase space during the microbunching instability. The proposed formula permits one also to link experimental information together, in particular the bunch length and the THz CSR spectrum.
\end{abstract}

DOI: 10.1103/PhysRevSTAB.17.010701

PACS numbers: 05.45.-a, 29.20.db, 41.60.Cr

\section{INTRODUCTION}

Contrary to the synchrotron radiation emitted by freeelectron lasers, the light emitted by electron bunches circulating in a storage ring is usually incoherent longitudinally, since there is no phase relation between emission by different electrons in a bunch. However, in special situations, emission can become coherent in the $\mathrm{THz}$ domain, and thus highly more intense. It happens when the electron bunch is shorter than the $\mathrm{THz}$ wavelength (typically, with a bunch length of few millimeters) [1] or when the longitudinal charge density has been modified by an external laser [2-4]. Beside these controlled situations, terahertz coherent synchrotron radiation (THz CSR) is also emitted spontaneously in storage rings when the number of electrons exceeds a threshold value, with the apparition of the so-called microbunching instability (e.g., [5-9]).

This instability is characterized by the presence of microstructures in the bunch, typically in the millimeters scale, thus associated with $\mathrm{THz}$ frequencies. It is usually a limitation for storage-ring operations; mainly because of the intense noise in the $\mathrm{THz}$ signal associated to an erratic behavior of the microstructures. However, when the $\mathrm{THz}$ signal fluctuations remain small, for example near the instability threshold, this instability can also be used as an intense $\mathrm{THz}$ source with a good signal-to-noise ratio [10].

*clement.evain@phlam.univ-lille1.fr

Published by the American Physical Society under the terms of the Creative Commons Attribution 3.0 License. Further distribution of this work must maintain attribution to the author(s) and the published articles title, journal citation, and DOI.
It is known, from numerical simulations [11] and stability analysis $[8,12]$, that the microstructures appear due to the interaction of the electrons with their own electric field (wakefield), in particular the so-called CSR wakefield emitted in bending magnets. However, little is known about the electron bunch characteristics during this instability, in particular how the electrons composing the bunch are distributed. The dynamics occurs essentially in a 2D phase space (longitudinal position $z$ and associated energy $E$ ), where direct experimental information is very difficult to obtain. Inversely, the $\mathrm{THz} \mathrm{CSR}$ emitted by one electron bunch is relatively easy to get, but is an indirect measurement of the 2D longitudinal phase space. Indeed, two projections are needed to get the value of the coherent $\mathrm{THz}$ signal $P_{\mathrm{THz}}$ from a 2D longitudinal electron distribution $f(z, E)$ (see Sec. II).

Here we show that the presence, in this coherent $\mathrm{THz}$ signal, of a frequency component in the radio-frequency (rf) domain gives precise information on the 2D phasespace structure of the electron bunch during the microbunching instability. This frequency, which we call $f_{m}$ ( $m$ for microstructures), has been observed in several storage rings (BESSY-II [13], MLS [14], SOLEIL [15], ANKA [16], and DIAMOND [17]). It is known to be a convenient diagnostic of the presence of microstructures (even small) in the bunch, and can be used for example to observe precisely the microbunching instability threshold [15].

We show here that this frequency $f_{m}$ is directly linked to the angle between the branches forming the patterns of the electron distribution. The value of this angle, obtained with a simple formula, is compared to the phase-space structure obtained by the numerical integration of the VlasovFokker-Planck (VFP) equation using the CSR wakefield. 
To test the validity of this work, two storage rings with very different parameters are considered, the Synchrotron SOLEIL and the facility UVSOR-II.

Moreover, thanks to a simple formula derived from the previous one, it is possible to link, with a rather good accuracy, information about the bunch length, the $\mathrm{THz}$ CSR spectrum and $f_{m}$. This can be used for example to estimate the value of $f_{m}$ in the case when this value is not known (from experiment or simulation).

\section{EQUATIONS AND PARAMETERS}

Numerical results are obtained by integrating the VlasovFokker-Planck (VFP) equation, with the shielded CSR wakefield [Eqs. (1) and (2)] [11] (using the opposite sign for the definition of $p$ compared to Ref. [11]). It is well known that this model (or the associated one using macroparticles) permits one to retrieve important characteristics of experimental $\mathrm{THz}$ CSR signal, in particular the modulation at $f_{m}$ [15] and at lower frequencies (oscillation or burst behavior) $[11,12]$.

The VFP equation describes the spatiotemporal evolution of $f(q, p, \tau)$, the phase-space density in the frame of the synchronous electron (the electron circulating along the circular orbit of radius $R$, with $R$ the bending magnet radius). $q, p, \tau$ are dimensionless variables, associated to $z$, $E$, and $t$ respectively $\left(\omega_{s}=2 \pi f_{s}, q=\frac{z}{\sigma_{z}}, \quad p=\frac{E-E_{0}}{\sigma_{E}}\right.$, $\tau=\omega_{s} \times t$, with $f_{s}$ the synchrotron frequency, $E_{0}$ the ring nominal energy, $\sigma_{z}$ and $\sigma_{E}$ the rms width, without collective effects, in position and in energy respectively). Without collective effects (i.e., without wakefields), the phase-space density is Gaussian in the two dimensions, and is rotating at the synchrotron frequency $f_{s}$ in phase space (due to an interplay between energy gain by rf cavities, energy losses by synchrotron radiation, and a path length depending on the electron energy). $\epsilon$ is the ratio between the damping time $\tau_{d}$ (due to synchrotron radiation) and $\omega_{s}\left(\epsilon=\frac{1}{\tau_{d} \omega_{s}}\right)$ :

$$
\begin{aligned}
& \frac{\partial f(q, p, \tau)}{\partial \tau}-p \frac{\partial f}{\partial q}+\frac{\partial f}{\partial p}\left(q-I_{c} E_{w f}\right) \\
& =2 \epsilon \frac{\partial}{\partial p}\left(p f+\frac{\partial f}{\partial p}\right) .
\end{aligned}
$$

The microstructures come from the interaction of the electrons with their own electric field $E_{w f}$, which is the convolution product of the longitudinal charge density $\rho(q)$ [where $\rho(q, \tau)=\int_{-\infty}^{+\infty} f(q, p, \tau) d p$ ] with the electric field of one electron. In this work, only the simplified model of the radiation of an electron circulating in an circular orbit (of radius $R$ ) between two infinitely conductive parallel plates (separated by an height $2 \times h$ ) is taken into account [Eq. (2)]. $I_{c}$ is the normalized current, proportional to $N$, the number of electrons in the bunch $\left(I_{c}=\frac{N e}{T_{0}} \frac{e 2 \pi R}{\sigma_{E} \omega_{s} T_{0}}\right.$ with $e$ the electron charge and $T_{0}$ the revolution period of the reference particle). $\gamma=E_{0} / m c^{2}, m$ the electron mass, $c$ the velocity of light, and $\epsilon_{0}$ is the vacuum permittivity. The expressions of the function $w$ and $G_{2}$ are given by Eqs. (3.12) and (5.22b) of Ref. [18]. Numerical estimation of [Eq. (2)] is done following a method similar to the one used in [19], and details are given in the Appendix:

$$
\begin{aligned}
E_{w f}(q)= & \frac{4}{3} \frac{\gamma^{4} T_{0}}{4 \pi \epsilon_{0} R^{2}} \int_{-\infty}^{+\infty} d \zeta \rho(q-\zeta) w\left(\frac{3 \gamma^{3}}{2 R} \sigma_{z} \zeta\right) \\
& -\frac{T_{0}}{8 \pi \epsilon_{0} h^{2}} \int_{-\infty}^{+\infty} d \zeta \rho(q-\zeta) G_{2}\left(\frac{\sigma_{z}}{2 R(h / R)^{3 / 2}} \zeta\right)
\end{aligned}
$$

The recorded coherent THz signal $P_{\mathrm{CSR}}(\tau)$ corresponds to the CSR power emitted in the detector bandwidth $\left(\nu_{0}, \nu_{f}\right)$ [cf. Eqs. (3)-(5)]. $P_{1 e}(\nu)$ is the power emitted by one electron at the frequency $\nu$, and is equal to the real part of the impedance of one electron [20]:

$$
\begin{gathered}
P_{\mathrm{CSR}}(\tau)=\int_{\nu 0}^{\nu f} P_{\mathrm{CSR}}(\nu, \tau) d \nu, \\
P_{\mathrm{CSR}}(\nu, \tau)=P_{1 e}(\nu) N^{2}|\tilde{\rho}(\nu, \tau)|^{2}, \\
\tilde{\rho}(\nu, \tau)=\int_{-\infty}^{+\infty} \rho(q, \tau) e^{-i 2 \pi \nu q} d q .
\end{gathered}
$$

To test the validity of our work over a large range of parameters (in particular the frequency $f_{m}=10 \mathrm{kHz}$ for SOLEIL and $f_{m}=650 \mathrm{kHz}$ for UVSOR, see Sec. III), these equations have been integrated with two sets of very different parameters, given in Table I. The UVSOR case is studied in normal alpha mode, whereas the SOLEIL case is studied in a low-alpha configuration (with alpha, the momentum compaction factor, linking the variation of the electron position $q$ to its energy $p$ [20]). For

TABLE I. Parameters of SOLEIL and UVSOR II storage rings used in the numerical simulations.

\begin{tabular}{lccc}
\hline \hline Parameter & Unit & SOLEIL & UVSOR II \\
\hline Nominal energy $E_{0}$ & $\mathrm{MeV}$ & 2750 & 600 \\
Relative energy spread $\sigma_{E}$ & & $10^{-3}$ & $3.4 \times 10^{-4}$ \\
Bunch length $\sigma_{z}$ & $\mathrm{~mm}$ & 1.4 & 23.4 \\
Damping time $\tau_{d}$ & $\mathrm{~ms}$ & 3.3 & 19 \\
Synchrotron frequency $f_{s}$ & $\mathrm{kHz}$ & 1.47 & 19.4 \\
Vacuum chamber height $2 \times h$ & $\mathrm{~mm}$ & 25 & 38 \\
Bending radius $R$ & $\mathrm{~m}$ & 5.36 & 2.2 \\
Current $I$ & $\mathrm{~mA}$ & 0.3 & 40 \\
\hline \hline
\end{tabular}


SOLEIL, experimental $\mathrm{THz}$ signals are published for this configuration [15].

\section{ORIGIN OF THE RF FREQUENCY IN THE THZ COHERENT SYNCHROTRON RADIATION}

The modulation of the $\mathrm{THz}$ signal in the rf domain is due to the spatiotemporal evolution of the microstructures in the 2D longitudinal phase space. The presence of microstructures in the phase space [Figs. 1(a) and 1(b)] induces a modulation in the longitudinal charge distribution $\rho(z, t)$ [Figs. 1(c) and 1(d)]. With time, this modulation drifts along the longitudinal coordinates because the phase space
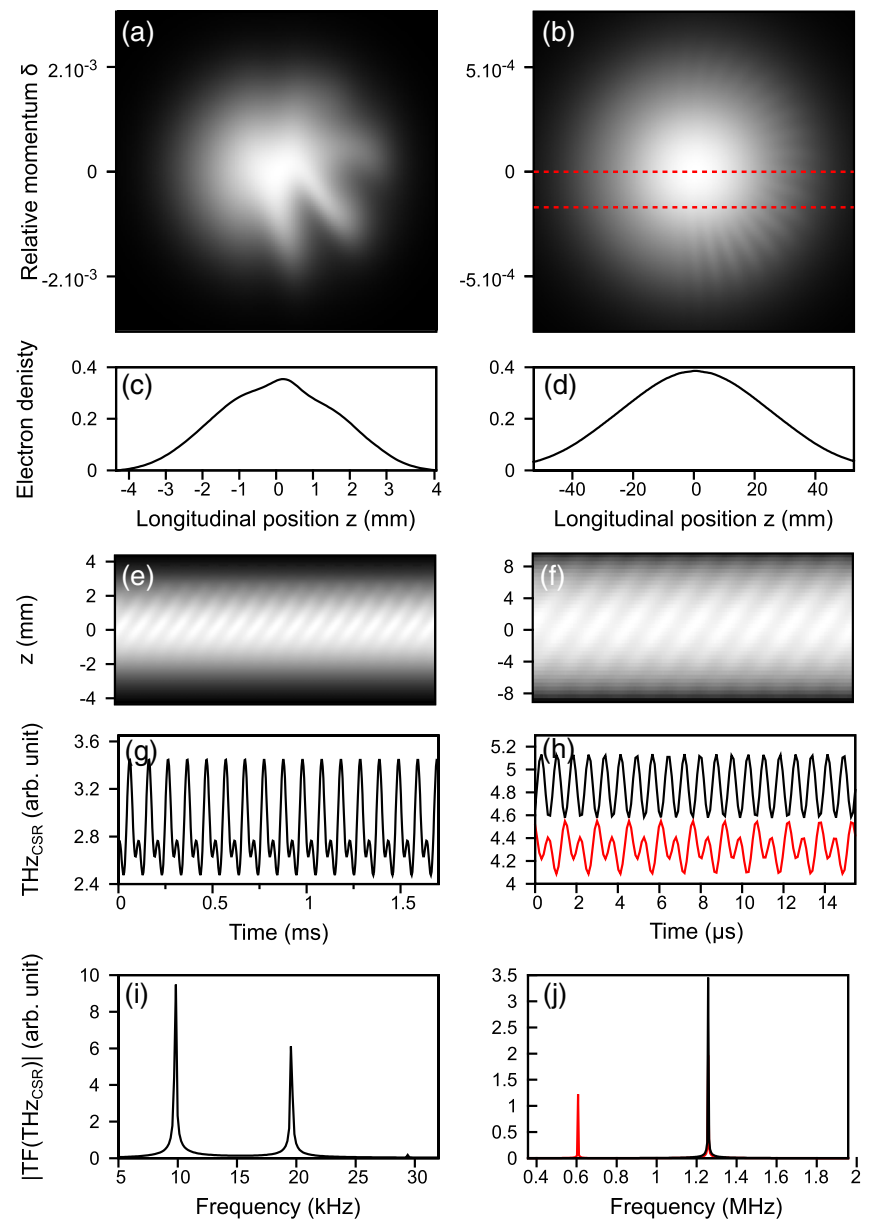

FIG. 1 (color online). From microstructures in the electronbunch longitudinal phase space $f(z, p, t)$ to modulation of the THz CSR power $P_{\mathrm{CSR}}(t)$. Numerical results with (a), (c), (e), (g), and (i) SOLEIL parameters and (b), (d), (f), (h), and (j) UVSOR II parameters. (a) and (b) Electron bunch phase space $f(z, p, 0)$ at the time $t=0$. (c) and (d) Associated longitudinal charge density $\rho(z, 0)$. (e) and (f) Time evolution of $\rho(z, t)$. (g) and (h) Associated $\mathrm{THz}$ power $P_{\mathrm{CSR}}(t)$. (i) and (j) Norm of the Fourier transform of $P_{\mathrm{CSR}}(t)$ : (black line) calculated from all the $2 \mathrm{D}$ phase-space distribution, (red line) calculated from the 2D distribution, where $-0.5<p<0$ (distribution between the red dashed lines in (b). In (h), the red line is shifted vertically for figure clarity. is rotating [Figs. 1(e) and 1(f)]. This behavior, coupled to the fact that the microstructures continuously appear in the bottom of the phase space, induces a modulation in the $\mathrm{THz}$ CSR signal [Figs. 1(g) and 1(h)]. As we can see on the Fourier transform of $P_{\mathrm{CSR}}(t)$ [Figs. 1(i) and 1(j)], this modulation is typically in the radio-frequency range, about $10 \mathrm{KHz}$ for SOLEIL and about $650 \mathrm{kHz}$ for UVSOR-II. For the UVSOR case, we observe that the peak at the second harmonic of $f_{m}$ is very dominant compared to the one at the fundamental frequency. The peak at the fundamental frequency can be nevertheless observed clearly if we record the CSR from only one part of the phase space [Figs. 1(b), 1(h), and 1(j), red curve].

\section{LINK BETWEEN ANGLE IN THE PHASE SPACE AND THE FREQUENCY MODULATION $f_{m}$ OF THE THZ SIGNAL}

Our goal here is to link the frequency $f_{m}$ of the THz CSR signal to the angle between branches forming the patterns in the 2D phase space. Our hypothesis is that the period $T_{m}$ $\left(T_{m}=1 / f_{m}\right)$ corresponds to the time needed to rotate by the angle $\varphi$, the angle between two branches. Since the angular velocity is $\omega_{s}=2 \pi f_{s}$, this implies the following relation between the angle $\varphi$ and $f_{m}$ :

$$
\varphi=\frac{2 \pi f_{s}}{f_{m}} .
$$

Using this relation with the parameters of Table I and the value of $f_{m}$ obtained with numerical simulations $\left[f_{m} \simeq\right.$ $10 \mathrm{kHz}$ for SOLEIL and $f_{m} \simeq 650 \mathrm{kHz}$ for UVSOR II, cf. Figs. 1(i) and 1(j)], we find $\varphi \simeq 53^{\circ}$ for SOLEIL and

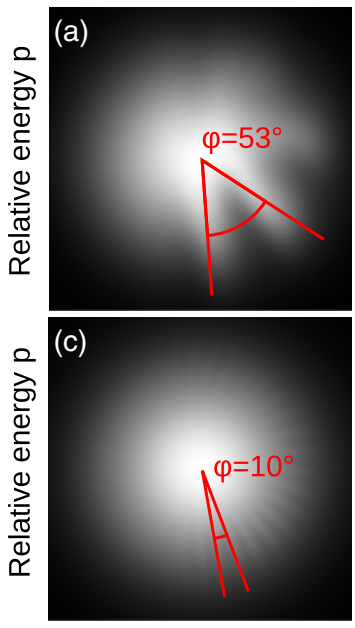

Longitudinal position q
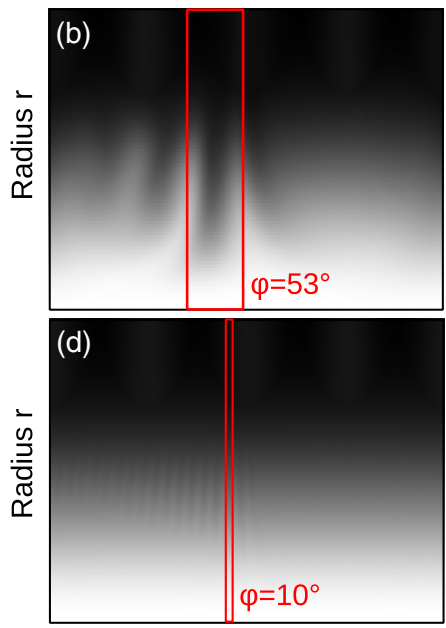

Angular position $\theta$
FIG. 2 (color online). Angle $\varphi$ calculated from the Eq. (6) (in red) superposed to the numerical phase space (in grey scale). (a) and (b) SOLEIL case and (c) and (d) UVSOR II case. (a) and (c) Cartesian $(q, p)$ coordinates and (b) and (d) polar $(r, \theta)$ coordinates (same parameters as Fig. 1). 
$\varphi \simeq 10^{\circ}$ for UVSOR II. As shown in Figs. 2(a) and 2(c), these angles are in good agreement with the angles between the branches of the 2D phase space density obtained by the numerical integration of the Vlasov-Fokker-Planck equation. This can also be observed in polar coordinates $r, \theta$ (with $q=r \times \cos \theta, p=r \times \sin \theta$ ) [Figs. 2(b) and 2(d)]. We predict that the relation (6) is all the more accurate since the phase-space structure keeps a similar shape during the rotation, so that the angle $\varphi$ does not change significantly with time. This implies that the relation (6) is more accurate near the instability threshold than far above the threshold, when the phase-space distortions are important (see for example Fig. 7 in [12]). It implies also that this relation is more accurate for systems with a low $\epsilon$ (i.e., a high value of the product damping time by synchrotron frequency); thus more accurate for UVSOR-II $\left(\epsilon=4.3 \times 10^{-4}\right)$ than for SOLEIL in low-alpha configuration $\left(\epsilon=3.3 \times 10^{-2}\right)$.

\section{LINK BETWEEN BUNCH LENGTH, THZ CSR SPECTRUM, AND $f_{m}$}

It is also possible to estimate the angle $\varphi$ with another formula, using the bunch length $\sigma_{z}$ and the THz CSR average spectrum $\left\langle P_{\mathrm{CSR}}(\nu, \tau)\right\rangle$ (the brackets denote averaging over the time $\tau)$ :

$$
\varphi \simeq \lambda_{\mathrm{CSR} \text { peak }} / \sigma_{z}
$$

with $\lambda_{\text {CSR peak }}$, the wavelength associated to the peak in $\left\langle P_{\mathrm{CSR}}(\nu, \tau)\right\rangle$ (cf. Fig. 4). This equation describes that at a distance $\sigma_{z}$ from the center of the phase space, the microstructure wavelength is about $\lambda_{\text {CSR peak }}$ (cf. Fig. 3).

The two expressions of $\varphi$ permit one to link the frequency $f_{m}$ with $\sigma_{z}$ and $\lambda_{\text {CSR peak }}$ :

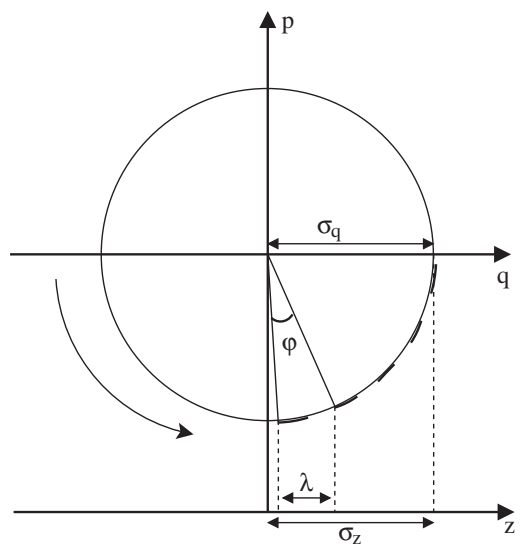

FIG. 3. Schematic representation of an electron-bunch phase space with a modulation located at a distance $\sigma_{q}$ from the bunch center. The distance $\lambda$ is associated to the projection on the horizontal axis (in unit of meters) of the arclength corresponding to one modulation period of the phase space. The distance $\lambda$ is generally associated to a coherent emission, and thus can be measured experimentally.
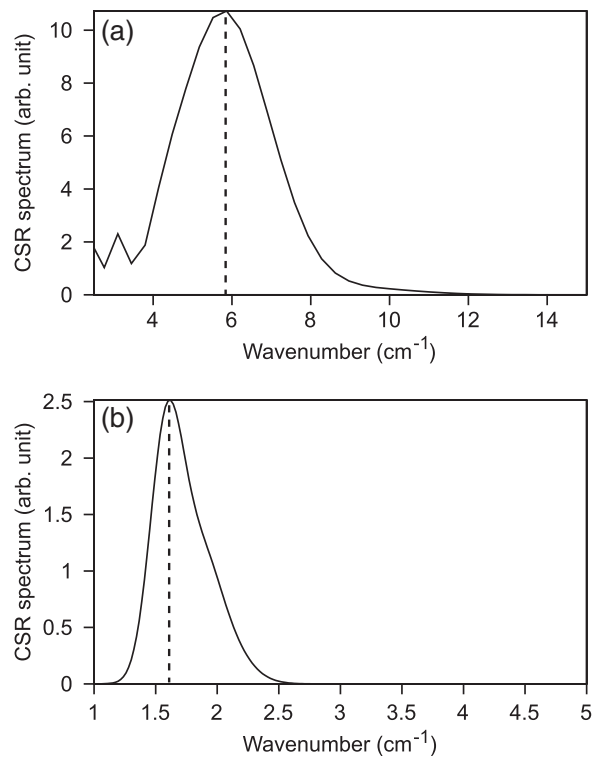

FIG. 4. Average THz CSR spectrum $\left\langle P_{\mathrm{CSR}}(\nu, \tau)\right\rangle$ in the case of (a) SOLEIL and (b) UVSOR-II. Vertical lines indicate $\lambda_{\text {CSR peak }}$ (same parameters as in Fig. 1).

$$
f_{m} \lambda_{\mathrm{CSR} \text { peak }} \simeq 2 \pi f_{s} \sigma_{z}
$$

In the case where only one of the parameters of Eq. (8) is not known, it is then possible to estimate it. Indeed, all the parameters in this equation can be obtained experimentally, but with very different techniques. $\sigma_{z}$ can be measured with a resolution of several picoseconds with a streak camera or from the fluctuations of the incoherent radiation [21]. $\left\langle P_{\mathrm{CSR}}(\nu, \tau)\right\rangle$ can be obtained experimentally with a Michelson interferometer coupled to a bolometer, at the condition that the fluctuations of the $\mathrm{THz}$ signal remain sufficiently small. The temporal THz CSR signal permits one to know $f_{m}$, provided the resolution of the $\mathrm{THz}$ detector is good enough.

As an example, we compare the value of $f_{m}$ given by this equation, with the value obtained from numerical simulations. Numerical $\mathrm{THz}$ spectra $\left\langle P_{\mathrm{CSR}}(\nu, \tau)\right\rangle$ of SOLEIL and of UVSOR-II are presented in Fig. 4. For SOLEIL, $\nu_{\mathrm{CSR} \text { peak }}=1 / \lambda_{\mathrm{CSR} \text { peak }} \simeq 6 \mathrm{~cm}^{-1}$, and for UVSOR $\nu_{\text {CSR peak }} \simeq 1.5 \mathrm{~cm}^{-1}$. For the value of $\sigma_{z}$, we use the value given by the analytical formula (values in Table I), and thus without taking into account collective effects. Results are given in Table II for the case of SOLEIL and UVSOR-II. In both cases, the agreement between the value of $f_{m}$ obtained

TABLE II. Value of the modulation frequency $f_{m}$ of the $\mathrm{THz}$ signal obtained from numerical simulations and with Eq. (8).

\begin{tabular}{lccc}
\hline \hline & $f_{m}$ from simulations & $f_{m}$ from Eq. (8) & Ratio \\
\hline SOLEIL & $10 \mathrm{kHz}$ & $8 \mathrm{kHz}$ & 1.25 \\
UVSOR II & $650 \mathrm{kHz}$ & $428 \mathrm{kHz}$ & 1.5 \\
\hline \hline
\end{tabular}


with numerical simulations and with Eq. (8) is equal or better to a factor 1.5 .

\section{CONCLUSION}

Precise information on the structure of an electron-bunch longitudinal phase space during the microbunching instability can be obtained from a radio-frequency component in the coherent terahertz signal emitted during this instability. More precisely, the angle between branches forming the pattern can be deduced using a simple analytical formula. We propose also an estimation of this angle using the bunch length and the THz CSR spectrum, which permit one to deduce possible unknown experimental parameters.

\section{ACKNOWLEDGMENTS}

This work was supported by the French "Agence Nationale de la Recherche" (ANR) (Blanc 2010-042301).

\section{APPENDIX: NUMERICAL METHOD FOR THE COMPUTATION OF THE SHIELDED CSR WAKEFIELD}

We consider the shielded CSR wakefield $E_{w f}$ generated by an electron moving on a circular orbit in the midplane of two infinitely conductive parallel plates that are separated by a distance $2 \times h$. The wakefield is composed of two contributions: the free-space term $(w)$ and the parallel plates term $\left(G_{2}\right)$.

Concerning the free-space contribution, we have to compute the convolution of $w\left(\frac{3 \gamma^{3}}{2 R} \sigma_{z} q\right)$ and the electron density $\rho(q)$. For that, we use an integration by parts. First, we compute the fast Fourier transform (FFT) of $v(\mu)$ [the antiderivative of $w(\mu)$, with $\mu=\frac{3 \gamma^{3}}{2 R} \sigma_{z} q$ ] with a very fine mesh [typically 100 times finer than the mesh for $\rho(q)$ ] and multiply it by $i k$. The function $v(\mu)$ is given by

$$
\begin{aligned}
v(\mu)= & \frac{9}{16}\left\{-\frac{2}{\mu}+\frac{1}{\mu \lambda}\left[(\lambda+\mu)^{1 / 3}+(\lambda+\mu)^{-1 / 3}\right]\right. \\
& \left.+\frac{2}{\lambda}\left[(\lambda+\mu)^{2 / 3}-(\lambda+\mu)^{-2 / 3}\right]\right\}
\end{aligned}
$$

with $\lambda=\sqrt{\mu^{2}+1}$. But because it is near zero, the function $v(\mu)$ varies very quickly (at a scale much shorter than one mesh step), a special process before the FFT computation is needed to increase the order of the computation method. We evaluate the exact value of the integration of $v(\mu)$ near zero within a $\delta q$ step size of the fine mesh. After this preprocess, we perform the FFT of the array $v_{i}$ as follows:

$$
v_{0}=\frac{1}{\delta q} \int_{0}^{\delta q / 2} v\left(\frac{3 \gamma^{3}}{2 R} \sigma_{z} q\right) d q
$$

$$
v_{i}=v\left(\frac{3 \gamma^{3}}{2 R} \sigma_{z} i \delta q\right) \quad \text { for } i>0
$$

Concerning the parallel plates contribution, we have to compute the convolution of $G_{2}\left(\frac{\sigma_{z}}{2 R(h / R)^{3 / 2}} q\right)$ and the density $\rho(q)$. For that, we compute the FFT of $G_{2}(x)$ (with $\left.x=\frac{\sigma_{z}}{2 R(h / R)^{3 / 2}} q\right)$ using a very fine mesh. The function $G_{2}$ is given by

$$
G_{2}(x)=2 \sum_{k=1}^{\infty} \frac{(-1)^{k+1}}{k^{2}}\left[\frac{4 Y_{k}^{4}\left(3-Y_{k}^{4}\right)}{\left(1+Y_{k}^{4}\right)^{3}}\right],
$$

where $Y_{k}$ is a root of the equation

$$
\frac{x}{k^{3 / 2}}=\frac{Y_{k}^{4}-3}{6 Y_{k}} \text {. }
$$

To avoid border effects for long electron bunches, in the simulations, we sum $k$ up to 150 .

After computing the FFT of the two terms of the wakefield, we compute the FFT of $\rho(q)$, multiply it by the previous calculated arrays, and then compute the inverse FFT.

[1] F. Sannibale, J. M. Byrd, A. Loftsdóttir, M. Venturini, M. Abo-Bakr, J. Feikes, K. Holldack, P. Kuske, G. Wüstefeld, H.-W. Hübers, and R. Warnock, Phys. Rev. Lett. 93, 094801 (2004).

[2] J. M. Byrd, Z. Hao, M. C. Martin, D. S. Robin, F. Sannibale, R. W. Schoenlein, A. A. Zholents, and M. S. Zolotorev, Phys. Rev. Lett. 97, 074802 (2006).

[3] S. Bielawski, C. Evain, T. Hara, M. Hosaka, M. Katoh, S. Kimura, A. Mochihashi, M. Shimada, C. Szwaj, T. Takahashi, and Y. Takashima, Nat. Phys. 4, 390 (2008).

[4] M. Hosaka, N. Yamamoto, Y. Takashima, C. Szwaj, M. Le Parquier, C. Evain, S. Bielawski, M. Adachi, H. Zen, T. Tanikawa, S. Kimura, M. Katoh, M. Shimada, and T. Takahashi, Phys. Rev. ST Accel. Beams 16, 020701 (2013).

[5] G. L. Carr, S. L. Kramer, J. B. Murphy, R. Lobo, and D. B. Tanner, Nucl. Instrum. Methods Phys. Res., Sect. A 463, 387 (2001).

[6] M. Abo-Bakr, J. Feikes, K. Holldack, G. Wüstefeld, and H.-W. Hübers, Phys. Rev. Lett. 88, 254801 (2002).

[7] J. M. Byrd, W. P. Leemans, A. Loftsdottir, B. Marcelis, M. C. Martin, W. R. McKinney, F. Sannibale, T. Scarvie, and C. Steier, Phys. Rev. Lett. 89, 224801 (2002).

[8] G. Stupakov and S. Heifets, Phys. Rev. ST Accel. Beams 5, 054402 (2002).

[9] Y. Takashima, M. Katoh, M. Hosaka, A. Mochihashi, S.-I. Kimura, and T. Takahashi, Jpn. J. Appl. Phys. 44, L1131 (2005).

[10] J. Barros, C. Evain, L. Manceron, J.-B. Brubach, M.-A. Tordeux, P. Brunelle, L. Nadolski, A. Loulergue, M.-E. 
Couprie, S. Bielawski, C. Szwaj, and P. Roy, Rev. Sci. Instrum. 84, 033102 (2013).

[11] M. Venturini and R. Warnock, Phys. Rev. Lett. 89, 224802 (2002).

[12] M. Venturini, R. Warnock, R. Ruth, and J. A. Ellison, Phys. Rev. ST Accel. Beams 8, 014202 (2005).

[13] P. Kuske, in Proceedings of the 23rd Particle Accelerator Conference, Vancouver, Canada, 2009 (IEEE, Piscataway, NJ, 2009), p. 4682.

[14] J. Feikes, M. von Hartrott, M. Ries, P. Schmid, G. Wüstefeld, A. Hoehl, R. Klein, R. Müller, and G. Ulm, Phys. Rev. ST Accel. Beams 14, 030705 (2011).

[15] C. Evain, J. Barros, A. Loulergue, M. A. Tordeux, R. Nagaoka, M. Labat, L. Cassinari, G. Creff, L. Manceron, J. B. Brubach, P. Roy, and M. E. Couprie, Europhys. Lett. 98, 40006 (2012).

[16] V. Judin, N. Hiller, A. Hofmann, E. Huttel, B. Kehrer, M. Klein, S. Marsching, C. Meuter, A.-S. Mueller, M. Nasse,
M. Schuh, M. Schwarz, N. Smale, and M. Streichert, in Proceedings of the 3rd International Particle Accelerator Conference, New Orleans, Louisiana, USA, 2012 (IEEE, Piscataway, NJ, 2012), p. 1623.

[17] R. Bartolini, G. Cinque, I. Martin, G. Rehm, and C. Thomas, in Proceedings of the 2nd International Particle Accelerator Conference, San Sebastián, Spain (EPS-AG, Spain, 2011), p. 3050.

[18] J. B. Murphy, S. Krinsky, and R. L. Gluckstern, Part. Accel. 57, 9 (1996).

[19] J. Qiang, Comput. Phys. Commun. 181, 313 (2010).

[20] A. W. Chao, Physics of Collective Beam Instabilities in High Energy Accelerators (John Wiley \& Sons, Inc., New York, 1993)

[21] F. Sannibale, G. V. Stupakov, M. S. Zolotorev, D. Filippetto, and L. Jägerhofer, Phys. Rev. ST Accel. Beams 12, 032801 (2009). 